\title{
Hérnias Diafragmáticas Traumáticas com Lesão Pericárdica: Apresentação e Complicaçōes Específicas
}

\author{
${ }^{1}$ Fernando Spencer Netto, ${ }^{2}$ Tiago Cesar Mierzwa, ${ }^{3}$ Mariana Thalita Bertolin Silva, ${ }^{4}$ Luan Geraldo Ocaña de Oliveira \\ ${ }^{5}$ Djoney Rafael dos Santos, ${ }^{6}$ Jean Carlo Barbosa
}

\section{RESUMEN}

Introducción: Hernia diafragmática traumática (HDT) se puede evolucionar con diversas complicaciones. Pacientes con lesión asociada al pericárdico, presentan risco de evolucionar con complicaciones específicas que se pueden llevar al óbito.

Objetivo: Este estudio tiene como objetivo dos casos de HDT con extensión pericárdico, con presentación o complicación grave e específica.

Casos clínicos: Primer caso: Paciente masculino de 34 años fue víctima de una caída de nivel de 8 metros de altura. Tomografía computadorizada (TC) mostró herniación del estómago para la cavidad torácica, líquido intraperitoneal y fracturas de la pelvis y acetabular. Fue sometido a laparotomía exploratoria por inestabilidad hemodinámica. En el postoperatorio inmediato, se observó la saída de alto flujo sanguíneo por tubo torácico, necesitando acercamiento por toracotomía, siendo realizado ligación del vaso sangrante del pericárdico. Presentó Buena evolución postoperatorio y sigue en la asistencia. Segundo caso: Paciente masculino de 54 años fue víctima de atropellamiento por motocicleta. Tomografía computadorizada mostró herniación del estómago para el saco pericárdico. Desarrollo un cuadro clínico de tamponamiento cardíaco y fue sujeto a laparotomía, que mostró hernia diafragmática intrapericárdica, la cual fue reducida, con mejora hemodinámica inmediata. Sin embargo, el paciente evolucionó con disfunción de múltiple órganos y sistemas, y falleció, dos días después de la admisión.

Conclusiones: La HDT con extensión pericárdica puede tener presentación o complicaciones específicas, como tamponamiento pericárdico por vísceras abdominales y sangría del pericárdico, que pueden llevar a óbito si no identificado y tratada rápidamente.

Importancia clínica: Debido la alta morbilidad y mortalidad, el diagnóstico precoz de la lesión pericárdica asociada a la hernia diafragmática a través de imagen y mientras la cirugía es de gran importancia, permitiendo un enfoque dirigido, que se pueda mejorar el desenlace clínico del paciente.

\footnotetext{
${ }^{1}$ Professor, ${ }^{2,3,5}$ Resident, ${ }^{4,6}$ Academic

1,4,6 Department of Medicina, Universidade Estadual do Oeste do Paraná, Cascavel, Paraná, Brazil

2,3,5 Department of General Surgery, Universidade Estadual do Oeste do Paraná, Cascavel, Paraná, Brazil

Corresponding Author: Fernando Spencer Netto, Professor of Medicine, Department of Medicina, Universidade Estadual do Oeste do Paraná, Cascavel, Paraná, Brazil, Phone: +554530383498, e-mail: fspencernetto@gmail.com
}

Palabras claves: Hernia diafragmática traumática, Lesión pericárdica, Traumatismo contuso.

How to cite this article: Netto FS, Mierzwa TC, Bertolin Silva MT, de Oliveira LGO, dos Santos DR, Barbosa JC. Hérnias Diafragmáticas Traumáticas com Lesão Pericárdica: Apresentação e Complicaçōes Específicas. Panam J Trauma Crit Care Emerg Surg 2016;5(3):161-165.

Source of support: Nil

Conflict of interest: None

\section{ABSTRACT}

Introduction: Traumatic diaphragmatic hernia (TDH) may develop several complications. Pericardial injury increases the severity of this entity and may lead to specific presentation and complications, that require immediate attention to avoid significant morbidity and mortality.

Objective: This study aims to report two cases of traumatic diaphragmatic hernia with pericardial extension with specific presentation or complication.

Clinical Case: Case 1: 34-year-old male suffered a fall of level of 8 meters high. Computed tomography (CT) scan showed herniation of the stomach into the left chest cavity, intraperitoneal fluid and pelvis and acetabulum fractures. He underwent exploratory laparotomy due to hemodynamic instability. In the immediate postoperative period, high amount of bloody output through chest tube was observed, requiring thoracotomy. At surgery, ligation of a bleeding vessel of the pericardium was performed. He showed good postoperative evolution, being discharged three weeks later. Case 2: 54-yearoldmale was hit by a motorcycle. Computed tomography scan showed herniation of the stomach into the pericardial sac. He developed clinical signs of cardiac tamponade and underwent laparotomy, which showed intrapericardial diaphragmatic hernia. It was reduced with immediate, but transient hemodynamic improvement. However, the patient developed multiple organ dysfunction, and died 2 days after admission.

Conclusion: Traumatic diaphragmatic hernia (TDH) with pericardial extension may show specific presentation and complications, such as pericardial tamponade by abdominal viscera and pericardial bleeding that can cause death if not identified and treated quickly.

Clinical significance: Due to the high morbidity and mortality of pericardial injury associated to diaphragmatic hernia, its early diagnosis through imaging or surgery has great importance, enabling adequate surgical approach and may improve the clinical outcome of the patients.

Keywords: Blunt trauma, Pericardial injury, Traumatic diaphragmatic hernia. 


\section{INTRODUÇÃO}

A hérnia diafragmática traumática (HDT) está presente em até $5 \%$ dos pacientes vitimas de trauma contuso severo admitidos no hospital, sendo essa lesão indicadora de trauma grave. ${ }^{1}$ A hérnia diafragmática traumática extensão pericárdica (HDTEP) é considerada uma complicação ou marcador de gravidade da HDT. Embora rara, se não diagnosticada e tratada rapidamente pode apresentar elevada taxa de mortalidade. ${ }^{2,3}$

A detecção e o diagnóstico precoce da HDT e HDTEP ainda representam um desafio médico. ${ }^{1}$ Além da dificuldade diagnóstica, podem evoluir rapidamente com graves complicações específicas, aumentando mortalidade. ${ }^{1,4,5}$

Este estudo tem como objetivo apresentar dois relatos de casos de HDTEP com complicações específicas graves e potencialmente fatais e realizar uma breve revisão sobre o tópico.

\section{CASO CLÍNICO}

\section{Primeiro caso}

Paciente masculino de 34 anos foi encaminhado ao pronto socorro de um serviço referência regional em trauma, vítima de queda de nível de 8 metros de altura. $\mathrm{Na}$ admissão, apresentava-se com via aérea definitiva por tubo orotraqueal e colar cervical. O exame clínico mostrou frequência cardíaca de $100 \mathrm{bpm}$, pressão arterial de 100/70 mmHg, saturação de oxigênio 97\%, expansibilidade torácica diminuída, timpanismo à percussão e murmúrio vesicular abolido em hemitórax esquerdo. Apresentava ainda abdome tenso e distendido, com presença de sangramento uretral.

Após a avaliação inicial seguindo princípios do ATLS, o paciente manteve-se estável hemodinamicamente e foi encaminhado para a realização de tomografia computadorizada (TC). A TC de tórax e abdome demonstrou hérnia diafragmática esquerda, com herniação do estômago para o interior da cavidade torácica (Figuras 1A to $C)$, associado a presença de líquido livre na cavidade abdominal, fraturas de acetábulo e ramo púbico esquerdo. A uretrocistografia retrógrada evidenciou lesão da uretra prostática.

Devido ao quadro clínico descrito, o paciente foi submetido à laparotomia exploradora. No intraoperatório, observou-se moderada quantidade de sangue coagulado na cavidade abdominal, com importante hematoma retroperitoneal de zona 3, lesão diafragmática extensa até pilar diafragmático, com cerca de 10 a $15 \mathrm{~cm}$, e presença de vísceras (estômago, omento e baço) na cavidade pleural. Assim, após redução das vísceras, observou-se sangramento esplênico difuso e discreto sangramento do diafragma. Realizadas esplenectomia, frenorrafia e drenagem pleural fechada à esquerda. Após término das rafias, o paciente não apresentava mais sangramento diafragmático. Em relação à lesão uretral, foi realizado cistostomia cirúrgica.

No pós-operatório, apresentou nas primeiras três horas, um débito de $1500 \mathrm{~mL}$ de sangue pelo dreno torácico. Foi optado pela reabordagem cirúrgica por toracotomia anterolateral esquerda, evidenciando grande quantidade de sangue vermelho vivo e coágulo na cavidade torácica, além de laceração importante em região do pilar diafragmático esquerdo com extensão até pericárdio, que apresentava sangramento ativo, não observado na laparotomia prévia. Foi realizada ligadura de sangramento arterial do pericárdio e fechada a incisão por planos, sendo hemotransfundido e encaminhado para a unidade de terapia intensiva (UTI). Após melhora clínica, foi encaminhado para enfermaria e recebeu alta hospitalar após 3 semanas. Atualmente, paciente permanece em acompanhamento ambulatorial, sem sinais de complicações pós operatórias.
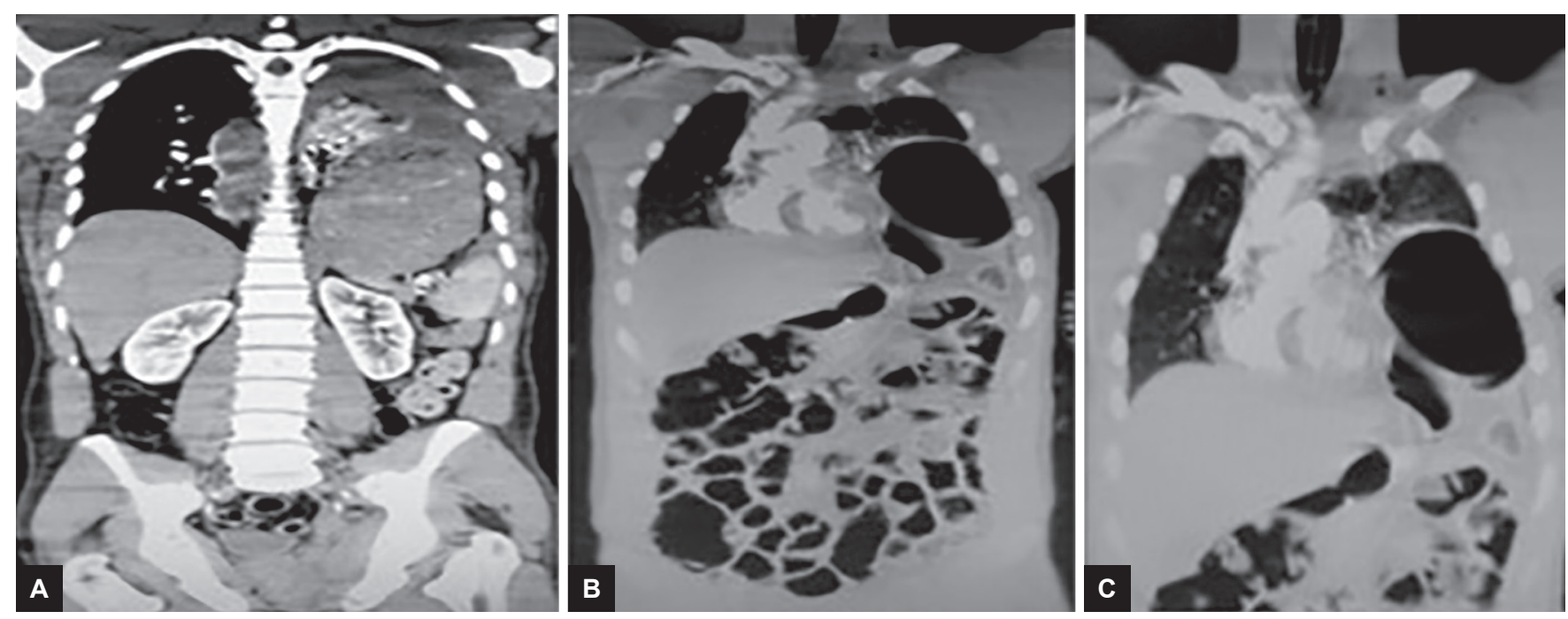

Figura 1A to C: Tomografia computadorizada toracoabdominal, pré-operatória, em corte coronal, evidenciando hérnia diafragmática à esquerda 


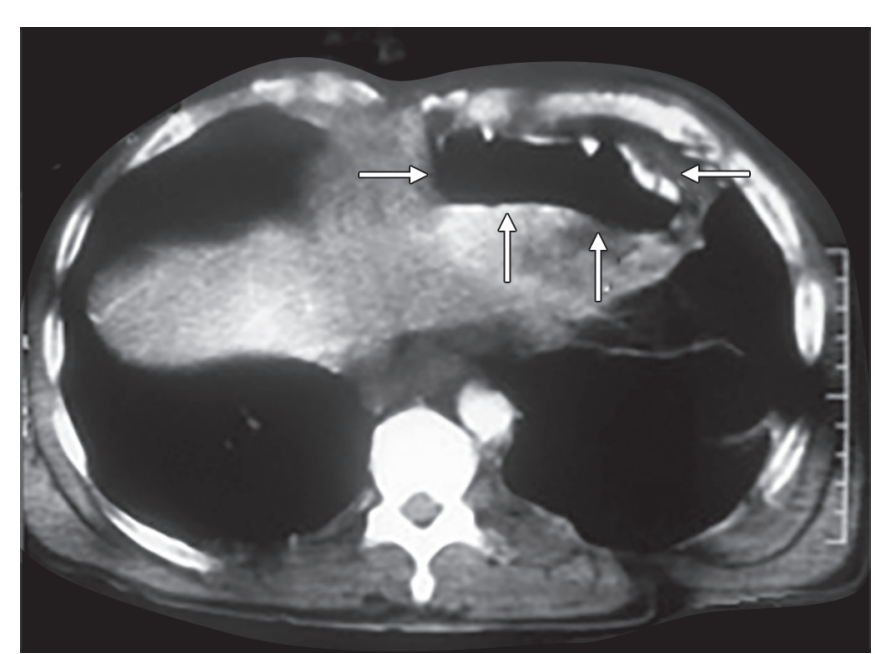

Figura 2: Tomografia computadorizada em corte sagital evidenciando a presença de bolha gástrica anterior ao coração. Observa-se a compressão das câmaras cardíacas direitas pelo estômago, resultando em um aspecto achatado

\section{Segundo caso}

Paciente masculino de 54 anos foi encaminhado ao pronto socorro de um serviço referência regional em trauma, vítima de atropelamento por motocicleta. $\mathrm{Na}$ admissão, o paciente chegou estável hemodinamicamente com imobilização em tábua rígida, colar cervical e tubo orotraqueal aplicados. Ao exame clínico, o paciente apresentava frequência cardíaca de 118 batimentos por minuto, pressão arterial de 108/56 $\mathrm{mmHg}$, bulhas cardíacas hipofonéticas, murmúrio vesicular presente e simétrico bilateralmente, assim como abdome discretamente distendido, porém flácido.

Após o manejo inicial, conforme o protocolo do ATLS, o paciente foi encaminhado para a realização TC de tórax e abdome que demonstrou contusão pulmonar, fraturas de $11^{\circ}$ e $12^{\circ}$ arcos costais esquerdos, pequena laceração esplênica, pequena quantidade de liquido na cavidade abdominal e fraturas dos ramos púbicos. A herniação do fundo gástrico para o interior do saco pericárdico (Figura 2) não foi percebida na avaliação inicial da TC.

Na sequência, foi encaminhado para a sala de emergência, e, após alguns minutos, evoluiu com hipotensão, turgência das veias jugulares e bulhas cardíacas hipofonéticas, com ausculta de ruídos hidroaéreos no foco mitral. TC foi revista e herniação gástrica para pericárdio detectada. Houve retardo de cerca de 2 horas entre a realização da TC, sua interpretação correta e encaminhamento para laparotomia de emergência, período no qual o paciente permaneceu hipotenso a despeito de infusão de volume e uso de drogas vasoativas. A cirurgia evidenciou hérnia intrapericárdica, que após redução, levou a melhora hemodinâmica imediata. Na sequência, foi realizado esplenectomia devido a lesão esplênica, frenorrafia, e drenagem torácica e do espaço pericárdico, sendo reparada a lesão do pericárdio diafragmático. Ainda no centro cirúrgico, foi realizada toracotomia ântero-lateral esquerda para diagnosticar possível lesão cardíaca, descartada após abertura e exploração do saco pericárdico. Ao fim da cirurgia, o paciente retornou a apresentar hipotensão, com necessidade de uso de drogas vasoativas.

No pós-operatório, o paciente permaneceu hemodinamicamente instável, anúrico, com disfunção de múltiplos órgãos. Evoluiu para óbito após 2 dias em UTI.

\section{DISCUSSÃO}

A ruptura diafragmática após trauma é uma condição pouco frequente, com incidência de 0,16 a $5 \%$ dos pacientes vítimas de trauma fechado. ${ }^{6} \mathrm{O}$ principal local acometido é o lado esquerdo, respondendo por $90 \%$ dos casos tratados em hospital, seguido do acometimento bilateral em $2 \%$ dos pacientes. ${ }^{1,5,6}$ A taxa de mortalidade varia de 5,5 a 51\%, ${ }^{6}$ decorrentes principalmente de choque hemodinâmico, falência de múltiplos órgãos e lesões cerebrais. ${ }^{1}$

A ocorrência de HDTEP é rara, e com frequência é diagnosticada apenas em autópsias. ${ }^{2,3}$ Esse tipo de lesão pode ocorrer em três regiões principais, na região diafragmática, com acometimento inferior do pericárdio, na região mediastinal superiormente e nas regiões pleuropericárdicas direita e esquerda. ${ }^{2}$

Segundo Kamiyoshihara M et al, em pacientes vítimas de trauma fechado, apenas $0,08 \%$ apresentaram ruptura pericárdica na região pleural e $0,03 \%$ apresentaram herniação cardíaca para a cavidade pleural. ${ }^{7}$ No presente relato, um dos pacientes apresentou lesão pericárdica com sangramento ativo e o outro evoluiu com herniação de conteúdo abdominal para o interior do saco pericárdico, levando a quadro clínico de tamponamento cardíaco.

Em geral, pacientes com HDT apresentam dor epigástrica, expansibilidade torácica diminuída, murmúrio vesicular abolido ou diminuído, presença de ruídos adventícios, bulhas cardíacas hipofonéticas, cavidade abdominal distendida, assimetria hipocondrial, ausculta de ruídos hidroaéreos na cavidade torácica e cianose, dispnéia, assim, como colapso hemodinâmico. ${ }^{5,8}$ Se o pericárdio pleural ou diafragmático forem lesionados, o coração pode herniar para o interior do espaço pleural e ocasionar constrição cardíaca, estrangulamento, torção total dos grandes vasos, ou herniação de conteúdo abdominal para o pericárdio, afetando significativamente hemodinâmica e podendo evoluir para óbito. ${ }^{3}$ Nos casos relatados, os pacientes apresentavam alguns desses sintomas, como murmúrio vesicular abolido, redução da expansibilidade torácica e sinais de tamponamento cardíaco (bulhas diminuídas, com hipotensão). Ambos evoluíram com instabilidade hemodinâmica após estabilidade inicial na admissão.

O diagnóstico clínico é muitas vezes difícil, sendo necessário o uso de exames de imagem, como radiografia de tórax, ultrassonografia de abdome, TC de tórax e 
abdome, e ressonância nuclear magnética em casos de exceção. ${ }^{4,5}$ Dentre esses, a TC é o método de escolha, com sensibilidade que varia de 61 to $87 \%$ e especificidade de 72 to $100 \%{ }^{6}$ O diagnóstico tardio de HDT pode levar a complicações graves. ${ }^{4}$

Diante de pacientes com suspeita de HDT, na avalição da TC deve-se procurar: sinais diretos de ruptura diafragmática, como descontinuidade diafragmática e deslocamento do diafragma, sinais indiretos que consistem na herniação de vísceras para a cavidade torácica, e na presença do sinal do colar, que corresponde a constrição focal de um segmento do tubo digestivo ou do omento através do orifício diafragmático. ${ }^{4}$ Além disso, a presença de imagens hipoatenuadas e extravasamento de contraste em região peridiafragmática, embora pouco específicas, podem auxiliar no diagnóstico. ${ }^{4,6}$ Quando suspeitamos de HDTEP, devemos avaliar a presença de descontinuidade diafragmática próximo ao pericárdio, presença de liquido ou vísceras no pericárdio. ${ }^{4,6}$ No segundo caso apresentado, o retardo entre a realização da TC e deteç̧ão da HDTEP provavelmente contribuiu para o óbito do paciente.

Outros métodos diagnósticos são a laparoscopia e a toracoscopia, que embora invasivos, permitem o diagnóstico e o tratamento concomitante dessa entidade. ${ }^{5,8}$ Sua utilização demanda estabilidade hemodinâmica e experiência da equipe. ${ }^{5}$ Embora existam diversos métodos para auxiliar no diagnóstico, apenas $43,5 \%$ dos casos de ruptura diafragmática são diagnosticados no período pré-operatório, enquanto que $41,4 \%$ são diagnosticados no intraoperatório ou na autópsia, e 14,6\% tardiamente após a cirurgia. ${ }^{3,9}$

Uma vez diagnosticado ou suspeito, o tratamento cirúrgico deve ser realizado o mais breve possível, sendo a abordagem por via abdominal considerada padrão ouro para os casos agudos de HDT. A abordagem pode ser laparotômica ou laparoscópica, indicada conforme a disponibilidade do serviço, condições clínicas do paciente e treinamento do cirurgião. ${ }^{5,8}$ Devido à elevada morbidade e mortalidade da extensão pericárdica, esta deve ser procurada ativamente. A não detecção da lesão pericárdica no primeiro caso relatado levou a sangramento pós-operatório significativo e consequente reoperação.

Com relação ao manejo cirúrgico da HDTEP, devido à sua ocorrência incomum, não há recomendação de uma conduta terapêutica padrão, sendo descrito tanto acesso torácico quanto abdominal para esses pacientes. ${ }^{3}$ Pericardiocentese não é recomendada. Este procedimento está em desuso para pacientes traumatizados por sua baixa eficiência e elevada morbidade. No caso particular de pacientes com HDTEP, não alivia a compressão do coração por vísceras abdominais. Nos presentes relatos, foram realizados laparotomia e toracotomia ântero-lateral primária. A toracotomia, no primeiro caso, permitiu encontrar a lesão pericárdica sangrante, não visualizada através da laparotomia. No segundo caso, permitiu descartar lesão cardíaca associada, o que por vezes é possível ver pela abertura do pericárdio diafragmático.

As principais causas de morte em pacientes que apresentam ruptura diafragmática são traumatismo craniano, hemorragia intra-abdominal, hemorragia pélvica, sepse, falência de múltiplos órgãos e sangramento intratorácico. ${ }^{1}$ Com relação aos nossos pacientes, ambos apresentaram ruptura diafragmática, lesões esplênicas, fraturas de pelve e acometimento pericárdico. Um deles evoluiu com falência de múltiplos órgãos e óbito.

\section{CONCLUSÃO}

Conclui-se que a HDTEP é uma condição secundária a trauma toracoabdominal grave, potencialmente fatal, que tem apresentação e complicações específicas do acometimento pericárdico. No presente relato, foram apresentados dois casos ilustrativos de apresentação e complicação especificas (tamponamento cardíaco por herniação de vísceras abdominais e sangramento pericárdico pós-operatório) e potencialmente fatais. Portanto, a HDTEP deve ser considerada uma situação de gravidade, alertando o médico assistente para procurar seu diagnóstico nos exames e cirurgia, minimizando assim a morbimortalidade.

\section{SIGNIFICÂNCIA CLÍNICA}

Devido à alta morbimortalidade da HDT com extensão pericárdica, o reconhecimento da sua possível apresentação e complicação é de grande importância, pois alerta o cirurgião para busca ativa deste diagnóstico através de imagem e durante a cirurgia, a fim de prover tratamento precoce e efetivo, melhorando possivelmente o prognóstico desses pacientes.

\section{DECLARAÇÕES}

Não há fontes de recursos.

Declaro que foi obtido o termo de consentimento com diretor do hospital para acessar as informações dos prontuários e do banco de imagem.

Não há conflitos de interesse.

\section{REFERÊNCIAS}

1. Chughtai T, Ali S, Sharkey P, Lins M, Rizoli S. Update on managing diaphragmatic rupture in blunt trauma: a review of 208 consecutive cases. Can J Surg 2009 Jun; 52(3): $177-181$. 
2. Witkowski Z, Lasek J, Wujtewicz M, Stasiak M, Marks W, Kawecka A. Pericardiodiaphragmatic rupture and cardiac herniation after multiple blunt trauma: diagnostic and therapeutic difficulties. J Thorac Cardiovasc Surg 2005 Dec;130(6):e9-e10.

3. Kuy S, Juern J, Weigelt JA. Laparoscopic repair of a traumatic intrapericardial diaphragmatic Hernia. JSLS 2014 AprJun;18(2):333-337.

4. Panda A, Kumar A, Gamanagatti S, Patil A, Kumar S, Gupta A. Traumatic diaphragmatic injury: a review of CT signs and the difference between blunt and penetrating injury. Diagn Interv Radiol 2014 Mar-Apr;20(2):121-128.

5. Dwivedi S, Banode P, Gharde P, Bhatt M, Johrapurkar SR. Treating traumatic injuries of the diaphragm. J Emerg Trauma Shock 2010 Apr-Jun;3(2):173-176.
6. Kaur R, Prabhakar A, Kochhar S, Dalal U. Blunt traumatic diaphragmatic hernia: pictorial review of CT signs. Indian J Radiol Imaging 2015 Jul-Sep;25(3):226-232.

7. Kamiyoshihara M, Nagashima T, Ibe T, Takeyoshi I. Rupture of the diaphragm and pericardium with cardiac herniation after blunt chest trauma. Gen Thorac Cardiovasc Surg 2010 Jun;58(6):291-294.

8. Hwang SW, Kim HY, Byun JH. Management of Patients with Traumatic Rupture of the Diaphragm. Korean J Thorac Cardiovasc Surg 2011 Oct;44(5):348-354.

9. Porto AS, Oshiro Y, Pontes JCVD, Medeiros CGS, Nandes AM, Ovando LA. Hérnia intrapericárdica transdiafragmática em trauma contuso: relato de caso e revisão da literatura. Revist Bras Cir Cardiovasc 1998;13:60-70. 